\title{
Spatial stochasticity and non-continuum effects in gas flows
}

\author{
S. Kokou Dadzie* \\ Mechanical and Aeronautical Engineering, Glyndwr University, \\ Mold Road, Wrexham LL11 2AW, UK \\ Jason M. Reese ${ }^{\dagger}$ \\ Department of Mechanical and Aerospace Engineering, \\ University of Strathclyde, Glasgow G1 $1 X J$, UK
}

\begin{abstract}
We investigate the relationship between spatial stochasticity and non-continuum effects in gas flows. A kinetic model for a dilute gas is developed using strictly a stochastic molecular model reasoning, without primarily referring to either the Liouville or the Boltzmann equations for dilute gases. The kinetic equation, a stochastic version of the well-known deterministic Boltzmann equation for dilute gas, is then associated with a set of macroscopic equations for the case of a monatomic gas. Tests based on a heat conduction configuration and sound wave dispersion show that spatial stochasticity can explain some non-continuum effects seen in gases.
\end{abstract}

PACS numbers: 47.45.Ab; 51.10.+y; 05.20.Dd; 02.50.Fz; 47.40.-x; 05.20.Jj; 47.70.Nd; 47.35.Rs; 47.10.ad Keywords: Stochastic equation; Brownian motion; Gas kinetic equation; Mass/Volume diffusion; Sound wave propagation; Non-continuum flow; Rarefied gas dynamics; Statistical mechanics

\footnotetext{
*Electronic address: k.dadzie@glyndwr.ac.uk

$\dagger$ Electronic address: jason.reese@strath.ac.uk
} 


\section{INTRODUCTION}

The Boltzmann kinetic equation is the standard model for dilute gas flows. However, as this equation lacks an exact solution, rarefied gas models generally consist of approximate solutions [1]. Most of these approximate models are constructed so that they reproduce the Navier-Stokes-Fourier model of continuum fluid mechanics under relevant assumptions. These kinetic models generally predict weakly thermodynamic disequilibrium flows well. However, "in a gas in which finite departures from equilibrium are imposed by forces too strong or too rapid to be overcome by collisions, a satisfactory comparison between kinetic theory and experiments is much harder to achieve" [2].

The Boltzmann kinetic model states an evolution equation of gas media presumed from deterministic equations of molecular motions. Subsequently, most derivations of it start with the deterministic Liouville equation, which enforces conservation of the microscopic phase space probability density $[1,3]$. The main characteristics of the Boltzmann equation are: it contains a collision integral describing the exchange of momentum between molecules; position and velocity variables are treated as independent variables; finally, and importantly, it does not involve an explicitly obvious stochastic component in the spatial variable. However, a large number of gaseous molecules exchanging momentum and positions represents a perfect example at the kinetic level of a physical stochastic process in both position and velocity spaces $[4]$.

Recognizing the distinction between a physical region in hydrodynamics and a physical region in kinetic theory, Klimontovich introduced a length scale separation, with averaging over kinetic space/volume, to arrive at a generalized kinetic equation that, in fact, is just a stochastic version of the deterministic Boltzmann equation (i.e., the Boltzmann equation with an additional spatial diffusion term) [5]. Zimdhal showed the importance of the Klimontovich version in deriving the relativistic Boltzmann equation [6]. Others, such as Ueyama [7], and Stryjewski et al [8], have also claimed a spatial stochastic term should be included in the deterministic Boltzmann kinetic model. Note that the stochastic Boltzmann equation is 
now known to derive from the more general stochastic Liouville equation $[9,10]$. While these works on stochastic Liouville and stochastic Boltzmann equations are mostly oriented toward other fields $[8,11]$, dilute gas flows remain predominantly the field of the deterministic Boltzmann equation. The various approximation solutions to the Boltzmann equation, and associated continuum equations, have their advantages and known limitations [12-14]. More precisely, the local-equilibrium assumption underlies most of these approximation models.

In this paper we develop a kinetic equation using strictly stochastic reasoning, without primarily referring directly to the Liouville or Boltzmann (deterministic) equations. The resulting kinetic equation, which is a stochastic version of the Boltzmann equation, is then associated with a set of macroscopic equations for the case of monatomic gases. The derivation is presented in a way that explicitly traces the impact of the additional spatial kinetic stochastic term on the macroscopic conservation equations of mass, momentum and energy. Predictions of thermodynamically non-equilibrium heat conduction and sound wave dispersion are then used to assess the role of the spatial stochastic terms.

\section{A STOCHASTIC KINETIC MODEL FOR GAS FLOWS}

\section{A. The Brownian model in position space}

Consider an arbitrary particle moving in physical space, with its position $X$ at a time t. This moving particle performs random walks in the sense that its trajectory is changing randomly. Then the problem is to obtain the spatial evolution in time of the particle, represented by a probability density function, $g(t, X)$. According to the Einstein-Smoluchowski model of Brownian motion, $g(t, X)$ satisfies the equation:

$$
\frac{\partial g}{\partial t}+\nabla \cdot J=0
$$

with $J$ given by,

$$
J=-\kappa \nabla g
$$


with $\kappa$ being a diffusivity coefficient. Equation (1) describes pure random movement, with no additional continuous displacement over time. If a smooth displacement, or drift motion, exists in addition to the pure random walk, then equation (1) becomes the Fokker-Planck model,

$$
\frac{\partial g}{\partial t}+(\xi \cdot \nabla) g+\nabla \cdot J=0
$$

where $\xi$ is the particle drift motion velocity.

Equation (3) is stochastic, in the sense that the third term on the left-hand-side, i.e. the spatial diffusion term, describes a spatial Brownian process [10]. Equation (3) can be used as a model for the spatial evolution of a targeted particle, where the focus is on the change in its position and trajectory due to the presence of a large number of other particles that obstruct its paths without transferring momentum to it $[4,9]$.

\section{B. The Brownian model in velocity space}

Consider now an arbitrary particle and focus on its velocity $\xi$ at a time $t$, while disregarding its position. We assume that this targeted particle is changing velocity in an uncontrollable way, and discontinuously. This is a random walk problem similar to the one in the previous section, but now in terms of velocity changes. So the problem is to obtain an evolution of the velocity distribution in time, described by a probability density function $h(t, \xi)$. A first attempt could be to use the Einstein-Smoluchowski equation (1) but substituting variable $\xi$ for $X[10]$. However, as the velocity variable (as opposed to the position variable) is related to the momentum and energy of the particle, a more representative Brownian model should be used for the evolution of the velocity distribution (see also the Appendix on why a Laplacian operator may not be a good velocity space Brownian model).

Let us represent the change in velocity or momentum of the targeted particle more generally by an operator, denoted $I_{\xi}(h(\xi))$, so that an equation for the velocity probability density reads:

$$
\frac{\partial h}{\partial t}-I_{\xi}(h(\xi))=0 .
$$


No particular assumption is made about the form of the operator $I_{\xi}(h(\xi))$, which remains just a general Brownian model operator at this point [15].

Rather than detailing the form of this operator, we wish to impose instead a certain number of requirements that it has to satisfy, based on the physical process driving velocity or momentum changes. For the case of hard-sphere particles, and assuming that the targeted particle changes its momentum in a way similar to elastic collisions between two solid bodies, the classical dynamic laws of collisions allow us to make the conditions:

$$
\int M I_{\xi}(h(\xi)) d_{\xi}=\int M \xi I_{\xi}(h(\xi)) d_{\xi}=\int M \xi^{2} I_{\xi}(h(\xi)) d_{\xi}=0
$$

that is, mass, momentum and energy are invariant under the velocity transformation represented by $I_{\xi}(h(\xi))$, after summing over all possible velocity states. In equation (5), $M$ represents the mass of the particle.

Equations (4) with (5) are a model for the probability density of a Brownian process in velocity space without drift. If a drift motion is considered as resulting from an additional external force, then a full stochastic version of equation (4) is given by:

$$
\frac{\partial h}{\partial t}+\left(F_{e x t} \cdot \nabla_{\xi}\right) h-I_{\xi}(h(\xi))=0
$$

with $F_{\text {ext }}$ denoting the external body force and $\nabla_{\xi}$ the gradient operator in velocity space.

\section{A stochastic kinetic equation and its macroscopic equations for a monatomic} gas

Consider now the complete physical situation, where a targeted monatomic gaseous molecule, moving within a group of similar gaseous molecules, changes both its trajectory and velocity or momentum in a stochastic way. Taking the position and velocity variables as independent, an equation for the multi-random-variable probability density, $f(t, X, \xi)$, can be written directly by combining equations (3) and (6) into:

$$
\frac{\partial f}{\partial t}+\xi \cdot \nabla f+\nabla \cdot J+F_{e x t} \cdot \nabla_{\xi} f-I_{\xi}(f)=0
$$


or, after substituting equation (2),

$$
\frac{\partial f}{\partial t}+\xi \cdot \nabla f-\nabla \cdot \kappa \nabla f+F_{\text {ext }} \cdot \nabla_{\xi} f-I_{\xi}(f)=0
$$

Equation (8) is a model stochastic kinetic equation for monatomic gases in which there are microscopic diffusion processes in both position and velocity. In fact, equation (8) can be regarded as a Fokker-Planck equation written for both position and velocity with drift [10], where the diffusive velocity flux is given by an operator specified by conditions (5) rather than being associated with a gradient operator.

Next, we derive a continuum-fluid macroscopic model of gas flow from kinetic model (8) and then compare it with the conventional model in two flow configurations. Hereafter, we disregard the body force $F_{\text {ext }}$, although our analysis will remain general.

\section{Macroscopic flow properties}

Macroscopic mass-density $\rho(t, X)$ and macroscopic flow velocity $U(t, X)$ may be defined through,

$$
\rho=\int M f(t, X, \xi) d_{\xi}
$$

and

$$
\rho U=\int M \xi f(t, X, \xi) d_{\xi}
$$

Then the peculiar velocity corresponds to

$$
C=\xi-U
$$

so that internal energy $e_{i n}(t, X)$ and the macroscopic momentum and energy diffusion flux tensor and vector, $\mathbf{P}_{i j}(t, X)$ and $\mathbf{q}(t, X)$, respectively, can be associated via:

$$
\rho e_{i n}=\int \frac{1}{2} C^{2} f d_{\xi}, \quad \mathbf{P}_{i j}=\iint C_{i} C_{j} f d_{\xi}, \quad \mathbf{q}=\int \frac{1}{2} C^{2} C f d_{\xi} .
$$

In these definitions, which involve summing over the velocity space, it is assumed that at a position centered at $X$ one may find a large number of molecules, covering a wide 
range in the velocity space, and each satisfying, individually, equation (8). Therefore during this averaging process, variable $X$ loses its kinetic spatial variable meaning and becomes a continuum type of position variable. This averaging is also consistent with imposing, locally, the conservation conditions (5) for the large number of molecules in velocity space. However, regarding an individual molecule, in equation (8) the spatial diffusion term represents the way surrounding molecules affect its position, while the velocity diffusion represents the way surrounding molecules affect its momentum. Consequently, equation (8), although derived assuming the position variable is independent of the velocity variable, and using a single particle density distribution, does contain explicitly information on correlations between the molecules in both position and velocity space. The presence of an explicit spatial diffusion term, beside velocity diffusion, is due primarily to the fact that in the kinetic statistical treatment position is not regarded as the integral of velocity but an independent variable in its own right.

\section{A macroscopic continuum set of equations}

Multiplying the stochastic kinetic equation (8) by $M, M \xi, M \xi^{2} / 2$, and integrating over velocity space, gives, respectively:

\section{Mass-density}

$$
\frac{\partial \rho}{\partial t}+\nabla \cdot[\rho U \underline{-\kappa \nabla \rho}]=0
$$

\section{Momentum}

$$
\frac{\partial \rho U}{\partial t}+\nabla \cdot[\rho U U]+\nabla \cdot[p \mathbf{I}+\Pi] \underline{-\nabla \cdot[\kappa \nabla(\rho U)]}=0
$$

\section{Energy}

$$
\begin{aligned}
\frac{\partial}{\partial t}\left[\frac{1}{2} \rho U^{2}+\rho e_{i n}\right]+\nabla \cdot\left[\frac{1}{2} \rho U^{2} U+\rho e_{i n} U\right] & +\nabla \cdot[(p \mathbf{I}+\Pi) \cdot U]+\nabla \cdot[\mathbf{q}] \\
& -\nabla \cdot\left[\kappa \nabla\left(\frac{1}{2} \rho U^{2}+\rho e_{i n}\right)\right]=0
\end{aligned}
$$


where $\mathbf{I}$ is the identity matrix, $\Pi=\mathbf{P}-p \mathbf{I}$ with $p$ the kinetic pressure related to the internal energy by $3 p=2 \rho e_{i n}$. Compared with the conventional fluid dynamic set of conservation equations, new terms in the above set are underlined. These terms all have clear meaning from the derivation: the underlined term in the mass-density equation is a mass or volume diffusion component resulting from random spatial distribution of molecules; similarly, the underlined terms in the momentum and energy equations are, respectively, momentum and energy diffusion resulting from the same random change in positions at the microscopic level (not the random exchange of momentum). The shear stress and heat flux, which result from molecular level exchange of momentum and energy, can be given their Navier-Stokes and Fourier's Law expressions:

$$
\Pi=-\mu\left[\nabla U+(\nabla U)^{t r}\right]+\eta \nabla \cdot U \mathbf{I} \text { and } \mathbf{q}=-\kappa_{h} \nabla T
$$

with $\mu / \eta$ and $\kappa_{h}$ being, respectively, the dynamic/volume viscosity and heat conductivity. Temperature, $T$, is associated, according to the kinetic theory definition, with $e_{i n}=3 / 2 R T$, with $R$ being the specific gas constant.

\section{EFFECTS OF THE ADDITIONAL DISSIPATIVE MASS/VOLUME FLUX}

The main result of the approach followed in the preceding section is that there is now in the new kinetic equation a diffusive term related to spatial variables, which leads to additional diffusive terms in the macroscopic fluid equations. To investigate the effects of such dissipative terms, particularly the mass or volume diffusion term in the macroscopic density equation, we investigate two thermodynamically non-equilibrium fluid situations: pure heat conduction, and sound wave dispersion.

\section{A. Density profile in heat conduction between parallel plates}

For the sake of clarity, the traditional set of conservation equations in fluid mechanics are set down here: 


\section{Mass-density}

$$
\frac{\partial \rho}{\partial t}+\nabla \cdot[\rho U]=0
$$

\section{Momentum}

$$
\frac{\partial \rho U}{\partial t}+\nabla \cdot[\rho U U]+\nabla \cdot[p \mathbf{I}+\Pi]=0
$$

\section{Energy}

$$
\frac{\partial}{\partial t}\left[\frac{1}{2} \rho U^{2}+\rho e_{i n}\right]+\nabla \cdot\left[\frac{1}{2} \rho U^{2} U+\rho e_{i n} U\right]+\nabla \cdot[(p \mathbf{I}+\Pi) \cdot U]+\nabla \cdot[\mathbf{q}]=0,
$$

which are the Navier-Stokes-Fourier equations when closed with equations (16).

Consider one-dimensional steady-state pure heat conduction in a dilute gas between parallel plates, using equations (17)-(19). As $U=0$ and no shear stress is involved in this situation, this set of equations reduces to:

$$
\begin{gathered}
\nabla \cdot[p \mathbf{I}]=0, \\
\nabla \cdot[\mathbf{q}]=0 .
\end{gathered}
$$

So, momentum equation (20) provides a constant pressure, while energy equation (21), if conductivity is assumed constant, will provide a linear temperature profile. Note that density equation (17) vanishes when $U=0$, so no information on the density is given by the set of equations (17)-(19).

The gas local-equilibrium equation of state could be invoked in an attempt to address this density profile problem; but a linear temperature profile in $p=\rho R T$ leads to a hyperbolic density profile as the pressure is kept constant. However, in heat conduction in the continuum-fluid limit it is generally admitted that the density is constant. Consequently, the solution of the conventional set of equations (17)-(19), along with an equation of state, appears in contradiction with the expected solution. Furthermore, experiments on a rarefied gas confined between two parallel plates indicate that density profiles are linear to a first approximation [16]. 
Now consider instead this one-dimensional steady-state heat conduction problem using our new set of equations (13)-(15). This set of equations reduces to:

$$
\begin{aligned}
\nabla \cdot \kappa \nabla \rho & =0, \\
\nabla p & =0, \\
\nabla \cdot[\mathbf{q}]-\nabla \cdot\left[\kappa \nabla \rho e_{i n}\right] & =0 .
\end{aligned}
$$

So, the momentum equation provides again a constant pressure for this configuration. With $3 p=2 \rho e_{i n}$, the solution set of equations (22)-(24) is equivalent to:

$$
\begin{array}{r}
\nabla \cdot \kappa \nabla \rho=0, \\
\nabla p=0, \\
\nabla \cdot[\mathbf{q}]=0 .
\end{array}
$$

This predicts simultaneously a linear density profile and a linear temperature profile (subject to constant conductivity coefficients), which is more in agreement with experimental observations $[16]$.

\section{B. Sound wave dispersion in rarefied gases}

Another compelling configuration that may be considered is the prediction of sound wave speed and damping in monatomic gases. In this case, the set of unsteady one-dimensional equations is considered, and linearized around the flow variables $\rho^{0}, T^{0}, p^{0}=R \rho^{0} T^{0}, U^{0}=0$. Perturbations around this ground state are given through:

$$
\begin{aligned}
& \rho=\rho^{0}\left(1+\rho^{*}\right), \quad T=T^{0}\left(1+T^{*}\right), \\
& U=U^{*} \sqrt{R T^{0}}, \quad p=p^{0}\left(1+p^{*}\right),
\end{aligned}
$$

where the asterisked variables represent dimensionless quantities. Linearizing $p=\rho R T$ gives $p^{*}=\rho^{*}+T^{*}$. The dimensionless space and time variables are given by,

$$
x=L x^{*}, \quad t=\frac{L}{\sqrt{R T^{0}}} t^{*}=\tau t^{*},
$$


with $\tau=L / \sqrt{R T^{0}}$ and $L$ a flow macroscopic length scale. The dimensionless linearized equations derived from (13)-(15), closed with equations (16), are then written:

\section{Mass-density}

$$
\frac{\partial \rho^{*}}{\partial t^{*}}+\frac{\partial U^{*}}{\partial x^{*}}-\kappa^{*} \frac{\partial^{2} \rho^{*}}{\partial x^{* 2}}=0
$$

\section{Momentum}

$$
\frac{\partial U^{*}}{\partial t^{*}}-\left(\frac{4}{3} \mu^{*}+\kappa^{*}\right) \frac{\partial^{2} U^{*}}{\partial x^{* 2}}+\frac{\partial \rho^{*}}{\partial x^{*}}+\frac{\partial T^{*}}{\partial x^{*}}=0
$$

\section{Energy}

$$
\frac{\partial T^{*}}{\partial t^{*}}+\frac{2}{3} \frac{\partial U^{*}}{\partial x^{*}}-\left(\kappa^{*}+\frac{2}{3} \kappa_{h}^{*}\right) \frac{\partial^{2} T^{*}}{\partial x^{* 2}}=0
$$

where the different dimensionless transport coefficients are given by:

$$
\mu=L \rho^{0} \sqrt{R T^{0}} \mu^{*}, \quad \kappa=L \sqrt{R T^{0}} \kappa^{*}, \quad \kappa_{h}=R \rho^{0} L \sqrt{R T^{0}} \kappa_{h}^{*} .
$$

Then the disturbances $\rho^{*}, T^{*}$ and $U^{*}$ are assumed to be wave functions of the form:

$$
\phi^{*}=\phi_{a}^{*} \exp \left[i\left(\omega t^{*}-K x^{*}\right)\right]
$$

where $\omega$ is the complex wave frequency, $K$ the complex wave number, and $\phi_{a}^{*}$ the complex amplitude, so that:

$$
\frac{\partial \phi^{*}}{\partial t^{*}}=i \omega \phi^{*}, \quad \frac{\partial \phi^{*}}{\partial x^{*}}=-i K \phi^{*}, \quad \frac{\partial^{2} \phi^{*}}{\partial x^{* 2}}=-K^{2} \phi^{*}, \quad \frac{\partial^{3} \phi^{*}}{\partial x^{* 3}}=i K^{3} \phi^{*} .
$$

The linearized hydrodynamic set of equations then yields the homogeneous system,

$$
\left(\begin{array}{ccc}
i \omega+\kappa^{*} K^{2} & 0 & -i K \\
0 & \left(\frac{2}{3} \kappa_{h}^{*}+\kappa^{*}\right) K^{2}+i \omega & -\frac{2}{3} i K \\
-i K & -i K & \left(\frac{4}{3} \mu^{*}+\kappa^{*}\right) K^{2}+i \omega
\end{array}\right)\left(\begin{array}{l}
\rho^{*} \\
T^{*} \\
U^{*}
\end{array}\right)=0 .
$$

The corresponding dispersion relation from the degeneracy requirement is then:

$$
\begin{array}{r}
\left(K^{2}\left(\frac{2 \kappa_{h}^{*}}{3}+\kappa^{*}\right)+i \omega\right)\left(2 i K^{2} \omega \kappa^{*}+\frac{4}{3} K^{4} \mu^{*} \kappa^{*}+K^{4} \kappa^{* 2}+\frac{4}{3} i K^{2} \omega \mu^{*}+K^{2}-\omega^{2}\right) \\
+\frac{2}{3} i K\left(K \omega-i K^{3} \kappa^{*}\right)=0 .
\end{array}
$$


Experimental data considered for comparison here are from Meyer and Sessler [17]. These experimental data are given in dimensionless form, using a dimensional analysis corresponding to the following expressions for dimensionless speed $\Upsilon$ and dimensionless damping coefficient $\Lambda$ :

$$
\frac{1}{\Upsilon}=\sqrt{\frac{5}{3}} \frac{\operatorname{Re}[K]}{\omega} \text { and } \Lambda=-\sqrt{\frac{5}{3}} \frac{\operatorname{Im}[K]}{\omega} .
$$

When expressing the speed and damping as in equations (37), sound modes are identified as harmonic pressure waves, and $\omega$ in that situation signifies a real positive dimensionless frequency and can also be a measure of Knudsen number and non-continuum behaviour [18]. Prandtl number is traditionally expressed as,

$$
P_{r}=\frac{\gamma}{\gamma-1} R \frac{\mu}{\kappa_{h}}=c_{p} \frac{\mu}{\kappa_{h}}
$$

Substituting equations (33) into (38) gives the Prandtl number in terms of the present dimensionless transport coefficients as:

$$
P_{r}=\frac{c_{p}}{R} \frac{\mu^{*}}{\kappa_{h}^{*}}=\frac{5}{2} \frac{\mu^{*}}{\kappa_{h}^{*}}
$$

using the monatomic value of $c_{p}=(5 / 2) R$. A monatomic gas has a Prandtl number of $2 / 3$ and an adiabatic exponent of $5 / 3$. To compare with experiments in this paper, we set $P_{r}=2 / 3, \kappa_{h}^{*}=1$, then from equation $(39), \mu^{*}=2 / 3 \times 2 / 5$ : in other words a combination of these coefficients giving the classical values of Prandtl number and adiabatic exponent, which in turn specifies also the sound speed in the continuum limit.

In our new stochastic continuum flow model, the value of the volume/mass diffusion coefficient $\kappa^{*}$ requires further investigation. Nevertheless, the Schmidt number and Lewis number are two known dimensionless numbers that can relate to a mass diffusion process:

$$
S_{c}=\frac{\mu}{\rho \kappa} \text { and } L_{e}=\frac{\kappa_{h}}{\rho c_{p} \kappa}
$$

or in terms of our dimensionless coefficients:

$$
S_{c}=\frac{\mu^{*}}{\kappa^{*}} \text { and } L_{e}=\frac{2}{5} \frac{\kappa_{h}^{*}}{\kappa^{*}} .
$$


Measurement data from [19] gives for $\mathrm{Ar} / \mathrm{CO}_{2}$ diffusion, which has a mass ratio of 1.1 , a value of $L_{e}=1.085$. As the species mass ratio is almost 1, that value of the Lewis number can be taken as a reasonable value for self-diffusion in Argon. Meanwhile, the value of $S_{c}$ indicated in the literature for a monatomic gas is $3 / 4$. Note that either the Lewis or the Schmidt number is required to specify $\kappa^{*}$ and solve the dispersion relation (36), but neither is involved in solving the dispersion relation that comes from the Navier-Stokes-Fourier model of equations. For our comparisons with experiment the two options of evaluating $\kappa^{*}$ via $S_{c}$ and $L_{e}$ have been investigated.

Figures 1 and 2 show, respectively, the predicted dimensionless damping coefficient and inverse dimensionless phase speed variation with inverse dimensionless wave frequency $\omega$, along with the argon gas experimental data from [17] and the predictions of the conventional Navier-Stokes-Fourier model. It is clear from the two figures that the new terms in the stochastic model produce an improvement in the predictions. The damping coefficient is usually the most difficult to predict [20], but figure 1 shows that our new stochastic model captures exactly the peak in the experimental prediction, and agrees with experiments well beyond $\omega=1$, while the Navier-Stokes-Fourier model fails already by $\omega \approx 0.2$. Our new model shows also a visible improvement in the prediction of the phase speed. It should be noted that the experimental data are believed to be doubtful only for $\omega>>1$, because of incompatibility of the experimental configuration with the definition of sound waves for large $\omega[2]$. However, the Navier-Stokes-Fourier model fails well before $\omega=1$. In contrast, the corrections introduced by the new stochastic model improve predictions usefully in the validity range of the experimental data for both the damping and speed.

In figures 1 and 2 the Prandtl number and adiabatic exponent of a monatomic gas have their conventional values; the dimensionless volume/mass diffusion coefficient is then given by $\kappa_{h}^{*} / \kappa^{*}=1.18$. This ratio is close enough to the experimental value of $L_{e}=1.085$ to suggest that it should correspond to the Lewis number from [19]. On the one hand, this appears to corroborate a previous suggestion that the volume/mass diffusivity coefficient should be equal to either the thermal diffusivity or the kinematic viscosity [21]. On the 


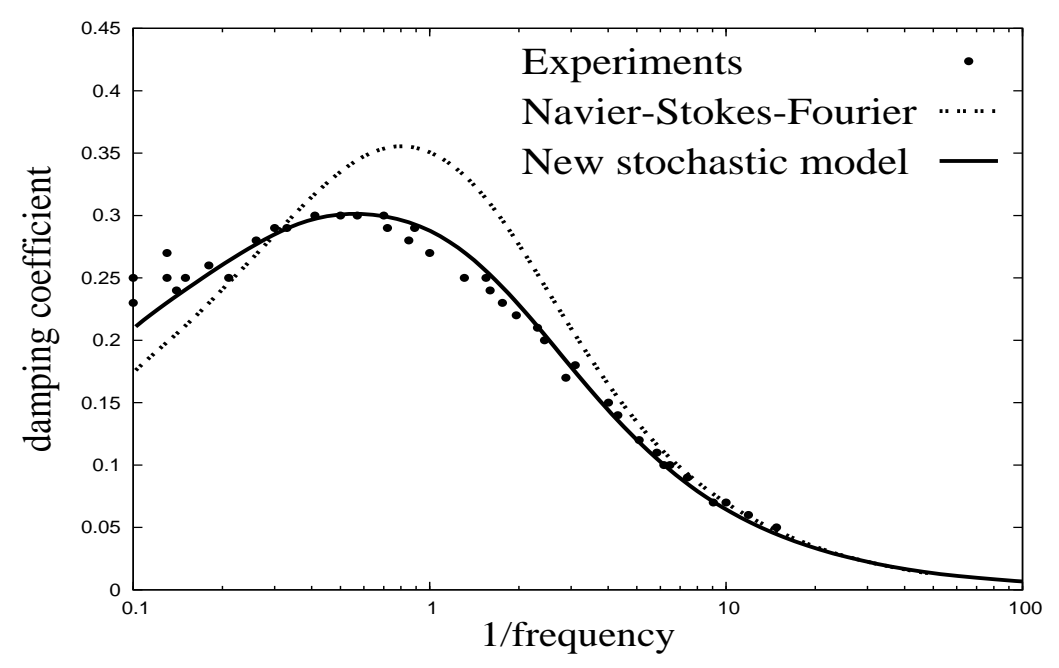

FIG. 1: Normalized damping coefficient varying with inverse dimensionless frequency.

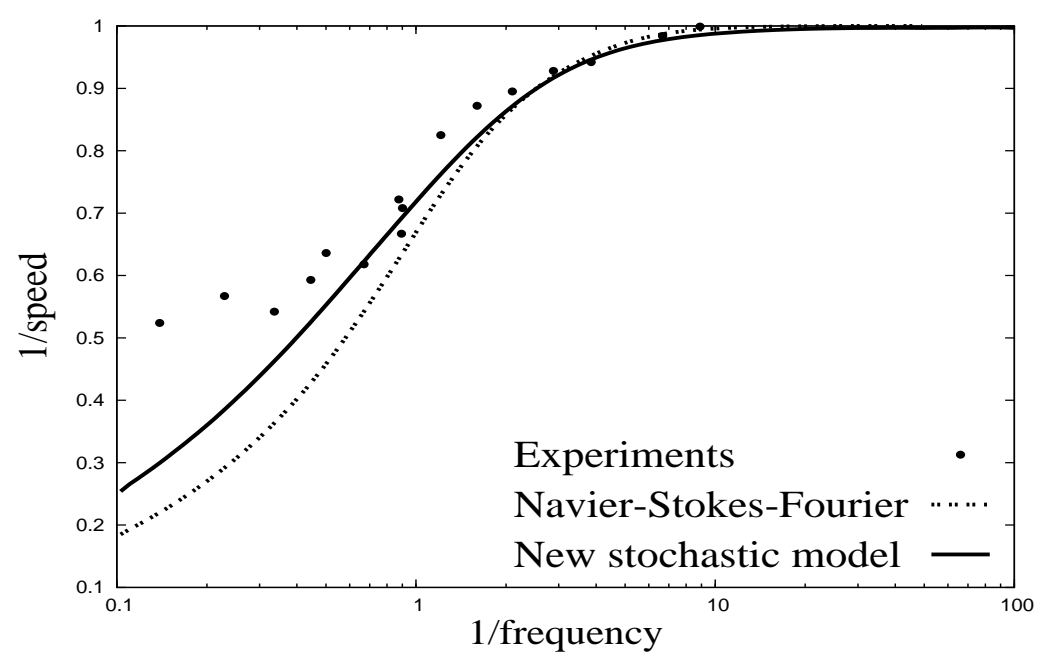

FIG. 2: Normalized inverse phase speed varying with inverse dimensionless frequency.

other hand, the conventional expression of the Lewis number given in (41) has an additional factor of $2 / 5$. This factor may be explained as follows: comparing energy equation (21) of the Navier-Stokes-Fourier model with our non-conventional model equation (24), the heat flux is affected by the mass/volume diffusion. As heat flux, and particularly heat conductivity, are conventionally measured without taking into account any mass/volume diffusion process or density/pressure gradient, it is possible that a disparity may exist in conductivity coefficients between the different continuum fluid models, which may affect 
the Prandtl or Lewis number. We found during our investigations that it is possible to get good agreement with the experimental data using a Schmidt number of 3/4, if the Prandtl number is reinterpreted to account for the mass/volume diffusion.

\section{CONCLUSION}

Two gas flow configurations have been investigated in the context of non-continuum and non-local-equilibrium behaviour. A stochastic kinetic model and associated set of continuum-fluid equations have been derived in order to assess the relationship between spatial stochasticity and non-continuum gas behaviour that the traditional Navier-StokesFourier model does not account for. In contrast to the conventional Boltzmann kinetic equation, our new kinetic equation incorporates a microscopic spatial diffusion, which leads to a volume/mass diffusion contribution to the macroscopic continuum-fluid equations. Our final continuum model is broadly similar in structure to those suggested by some previous investigators [5]. New results reported in this paper for the heat conduction and sound wave dispersion problems strengthen the connection between spatial stochasticity and noncontinuum effects $[18,22,23]$. However, we note that the existence of volume/mass diffusion in the continuum-fluid model is controversial with regard to macroscopic thermodynamics and some classical mechanics principles [24]. A thermodynamic framework consistent with a volume/mass diffusion continuum-fluid model is still to be clarified.

\section{Acknowledgements}

The authors would like to thank the Royal Academy of Engineering and the Leverhulme Trust, in the U.K. for funding through a Senior Research Fellowship that enabled the com- 
pletion of this research.

[1] C. Cercignani, Theory and Application of the Boltzmann Equation, Scottish Academic Press, 1975.

[2] F. S. Sherman, L. Talbot, Experiment versus kinetic theory for rarefied gases, in: von Karman, H. L. Dryden (Eds.), International Series on Aeronautical Sciences and Space Flight, Vol. 3, Rarefied Gas Dynamics, 1960, p. 161.

[3] J. M. Blatt, A. H. Opie, A new derivation of the Boltzmann transport equation, Journal of Physics A 7 (9) (1974) 1895-1906.

[4] P. Kotelenez, Stochastic Ordinary and Stochastic Partial Differential Equations, Springer, 2008.

[5] Y. L. Klimontovich, The unified description of kinetic and hydrodynamic processes in gases and plasmas, Physics Letters A 170 (6) (1992) 434-438.

[6] W. Zimdahl, Relativistic stochastic Boltzmann equation and fluctuations in general relativity, Classical and Quantum Gravity 6 (1989) 1879-1892.

[7] H. Ueyama, The stochastic Boltzmann equation and hydrodynamic fluctuations, Journal of Statistical Physics 22 (1) (1980) 1-26.

[8] J. Stryjewski, S. Ayikf, M. Beliacem, E. Suraud, A comparison of the velocity spectra obtained with the Boltzmann and Boltzmann-Langevin equations, in: International Workshop on Gross Properties of Nuclei and Nuclear Excitations, Hirschegg, Austria, 1990.

[9] R. Kubo, Stochastic Liouville equations, Journal of Mathematical Physics 4 (2) (1962) 174.

[10] S. Chandrasekhar, Stochastic problems in physics and astronomy, Review of Modern Physics $15(1)(1943) 1-89$.

[11] Y. Tanimura, Stochastic Liouville, Langevin, FokkerPlanck, and Master equation approaches to quantum dissipative systems, Journal of the Physical Society of Japan 75 (8) (2006) 082001.

[12] X.-J. Gu, D. R. Emerson, A high-order moment approach for capturing non-equilibrium phe- 
nomena in the transition regime, Journal of Fluid Mechanics 636 (2009) 177-216.

[13] R. K. Agarwal, K.-Y. Yun, R. Balakrishnan, Beyond Navier-Stokes: Burnett equations for flows in the continuum-transition regime, Physics of Fluids 13 (10) (2001) 3061.

[14] B.-Y. Cao, J. Sun, M. Chen, Z.-Y. Guo, Molecular momentum transport at fluid-solid interfaces in mems/nems, International Journal of Molecular Sciences 10 (11) (2009) 4638-4706.

[15] V. M. Kenkre, E. W. Montroll, M. F. Shlesinger, Generalized master equations for continuoustime random walks, Journal of Statistical Physics 9 (1) (1973) 45.

[16] W. P. Teagan, G. S. Springer, Heat transfer and density-distribution measurements between parallel plates in the transition regime, Physics of Fluids 11 (3) (1968) 497-506.

[17] E. Meyer, G. Sessler, Schallausbreitung in gasen bei hohen frequenzen und sehr niedrigen drucken, Zeitschrift fur Physik 149 (1957) 15-39.

[18] S. K. Dadzie, J. M. Reese, A volume-based hydrodynamic approach to sound wave propagation in a monatomic gas, Physics of Fluids 22 (1) (2010) 016103.

[19] J. Liu, G. Ahlers, Rayleigh-Bernard convection in binary-gas mixtures: Thermophysical properties and the onset of convection, Physical Review E 55 (6) (1997) 6950-6968.

[20] P. J. Dellar, Macroscopic descriptions of rarefied gases from the elimination of fast variables, Physics of Fluids, 19 (10) (2007) 107101.

[21] H. Brenner, Bi-velocity transport processes. single-component liquid and gaseous continua, Physica A 389 (2010) 389 (2010) 1297-1316.

[22] C. J. Greenshields, J. M. Reese, The structure of shock waves as a test of Brenner's modifications to the Navier-Stokes equations, Journal of Fluid Mechanics 580 (2007) 407-429.

[23] H. Brenner, A nonmolecular derivation of maxwells thermal-creep boundary condition in gases and liquids via application of the lechatelierbraun principle to maxwells thermal stress, Physics of Fluids 21 (053602) (2009) 21.

[24] H. C. Öttinger, H. Struchtrup, M. Liu, Inconsistency of a dissipative contribution to the mass flux in hydrodynamics, Physical Review E 80 (5) (2009) 056303.

[25] S. V. Bogomolov, L. W. Dorodnitsyn, Stochastic quasi gas dynamics equations. viscous gas 
case, Mathematical Models and Computer Simulations 3 (4) (2011) 457-467.

\section{Appendix}

In the Einstein-Smoluchowski equation (1), the Brownian operator is given by the spatial diffusion operator $(\nabla \cdot \nabla)$. Let us test this operator for representing velocity diffusion within the stochastic kinetic description of a monatomic gas, i.e:

$$
I_{\xi}(h)=\kappa_{\xi}\left(\nabla_{\xi} \cdot \nabla_{\xi}\right) h,
$$

where $\kappa_{\xi}$ is a kinetic velocity diffusivity coefficient and $\nabla_{\xi}$ is the gradient operator in velocity space. In this case we have,

$$
\int M I_{\xi}(h(\xi)) d_{\xi}=\int M \xi I_{\xi}(h(\xi)) d_{\xi}=0
$$

but

$$
\int M \xi^{2} I_{\xi}(h(\xi)) d_{\xi}=2 \kappa_{\xi} \rho
$$

This means that the diffusion operator (42) conserves, locally, mass and momentum but does not conserve energy. Consequently, it rules out the choice of this operator for diffusion in velocity or momentum space. The Boltzmann collision integral may be regarded as an example of a velocity-space Brownian operator; more generally, random walks can be modelled by various Brownian operators [15]. It is therefore, essential to follow consistent physical arguments when deciding on the correct operator to describe each microscopic exchange [25]. 